\title{
Nonresonant detection of Josephson radiation from thin-film microbridges
}

Sørensen, O. H.; Mygind, Jesper; Pedersen, Niels Falsig; Gubankov, V. N.; Levinsen, M. T.; Lindelof, Poul Erik

Published in:

Journal of Applied Physics

Link to article, DOI:

$10.1063 / 1.323542$

Publication date:

1977

Document Version

Publisher's PDF, also known as Version of record

Link back to DTU Orbit

Citation (APA):

Sørensen, O. H., Mygind, J., Pedersen, N. F., Gubankov, V. N., Levinsen, M. T., \& Lindelof, P. E. (1977).

Nonresonant detection of Josephson radiation from thin-film microbridges. Journal of Applied Physics, 48(12), 5372-5374. https://doi.org/10.1063/1.323542

\section{General rights}

Copyright and moral rights for the publications made accessible in the public portal are retained by the authors and/or other copyright owners and it is a condition of accessing publications that users recognise and abide by the legal requirements associated with these rights.

- Users may download and print one copy of any publication from the public portal for the purpose of private study or research.

- You may not further distribute the material or use it for any profit-making activity or commercial gain

- You may freely distribute the URL identifying the publication in the public portal

If you believe that this document breaches copyright please contact us providing details, and we will remove access to the work immediately and investigate your claim. 


\title{
Nonresonant detection of Josephson radiation from thin- film microbridges
}

\author{
O. H. Soerensen, J. Mygind, and N. F. Pedersen \\ Physics Laboratory I, The Technical University of Denmark, DK-2800 Lyngby, Denmark \\ V. N. Gubankov* \\ Institute of Radioengineering and Electronics of The Academy of Science of the USSR, Moscow K-9, USSR
}

M. T. Levinsen and P. E. Lindelof

Physics Laboratory I, H.C. Grsted Institute, University of Copenhagen, DK-2100 Copenhagen, Denmark (Received 29 November 1976; accepted for publication 8 February 1977)

\begin{abstract}
Measurements are reported of the Josephson radiation from microbridges coupled to an $X$-band receiver via a nonresonant microwave transformer. Although closely coupled to the bridge, the transformer does not modify the bridge properties. We find that near the transition temperature the radiation linewidth is proportional to the square of the dynamic resistance, which is also predicted by the resistively shunted junction model. The effective noise temperature determined by the linewidth is $19 \pm 3 \mathrm{~K}$ and the measured maximum integral power is $5 \times 10^{-12} \mathrm{~W}$.
\end{abstract}

PACS numbers: $74.50+\mathrm{r}, 85.25 .+\mathrm{k}, 73.40 . \mathrm{Jn}, 73.60 . \mathrm{Ka}$

The Josephson radiation from superconducting small capacitance weak links has been observed previously. Already Dayem and Grimes ${ }^{1}$ reported on microwave emission from point-contact junctions. Only recently similar results were obtained from variable-thickness bridges $^{2}$ and from cross-scratched microbridges. ${ }^{3}$ Common to these experiments was that the weak links were mounted in a microwave cavity $(Q \approx 500)$ in order to improve the matching of impedances between the weak link and the waveguide system. The presence of the cavity did, however, strongly modify the properties of the weak link due to the regenerative interaction between the active and highly nonlinear device and the cavity fields. As a result of this interaction the linewidth of the Josephson radiation was narrowed considerably, a phenomenon closely correlated to the presence of a cavity-induced step in the dc $I-V$ curve, and, furthermore, the results must be interpreted with care due to the existence of the so-called non-Josephson radiation ${ }^{4}$ encountered in resonant systems. Consequently, in any attempt to study the intrinsic microwave properties of weak links, such resonant interactions should be carefully avoided.

In this paper we report the first nonresonant detection of the Josephson radiation from superconducting mic robridges at $X$-band frequencies. The problem of impedance matching was solved using a broadband waveguide transformer. In this way a coupling between the mic robridge and the standard $X$-band waveguide system was realized which was about one order of magnitude better than previously obtained with similar samples mounted in resonant systems. ${ }^{2,3}$ Furthermore, the emitted power measured as a function of the dc voltage reproduced the spectral distribution of the Josephson radiation ${ }^{5}$ because of the absence of regenerative cavity interactions. Thus, we were able to measure properties

\footnotetext{
*Work performed during a stay at Physics Laboratory 1 , The Technical University of Denmark, and Physics Laboratory $I$, University of Copenhagen, Denmark.
}

of the autonomous junction and were for the first time able to test unambiguously the applicability of the resistively shunted junction (RSJ) model to our junctions.

The thin-film microbridges used in this experiment were cross scratched in a 2000 - $\AA$-thick indium film on a $12 \times 25-\mathrm{mm}^{2}$ glass substrate. ${ }^{6}$ The dimensions of the bridges were typically $0.5 \times 0.5 \mu \mathrm{m}^{2}$ and the normalstate resistance near $T_{c}$ typically was $0,15 \Omega$. The sample was mounted directly across the low-impedance end of the waveguide transformer section with a thin Mylar foil inserted for dc insulation. The transformer reduced the height of the standard $X$-band waveguide from 10.2 to $0.4 \mathrm{~mm}$ in two steps, each of length $\frac{1}{4} \lambda$. The bandwidth was measured to $600 \mathrm{MHz}$ centered at 9.0 $\mathrm{GHz}$ and the impedance matching the narrow section was calculated to $6.5 \Omega$. The microwave system was a conventional superheterodyne receiver with a measured overall noise figure of $9.5 \mathrm{~dB}$. The $i_{\text {。 }} f$. amplifier had a center frequency of $70 \mathrm{MHz}$ and a bandwidth of 10


lower receiver sideband was within the transformer bandwidth. The contribution to the signal from the upper sideband could in this way be suppressed by more than $20 \mathrm{~dB}$. Three isolators and a low-pass filter $\left(f_{\mathrm{c}} \approx 16\right.$ $\mathrm{GHz}$ ) prevented LO power from reaching the junction. Finally, a PIN diode modulator was inserted in the receiver input and the modulated videosignal synchronously detected in a lock-in amplifier.

Figure 1 shows the detected rf power and the $I-V$ curve at two different temperatures. The inset shows a typical recording of the differential resistance $d V / d I$ at a temperature close to $T_{c}$. Note that the broadband transformer does not cause structure in the $I-V$ curve or in the $d V / d I$ curve. The peaks of the radiation spectrum are centered at voltages $V_{n}=h \nu_{R} / 2 n e$ where $\nu_{R}$ is the receiver frequency and $n$ is an integer. At the temperatures closest to $T_{c}$ [Fig. 1 (a)] only radiation corresponding to the fundamental, $n=1$, was clearly resolved. At lower temperatures the $n=2$ and $n=3$ peaks 




FIG. 1. dc current and $\mathrm{xf}$ power as a function of dc voltage at two different temperatures. (a) $T=3.42 \mathrm{~K}$ and (b) $T=3.35 \mathrm{~K}$. The left- and right-hand vertical scales are for current and measured rf power, respectively. The inset in (a) shows the differential resistance $d V / d I$ at a temperature near $T_{c}$. LO frequency $=9.199 \mathrm{GHz} ; T_{c}=3.44 \mathrm{~K}$.

gradually appeared [Fig。 1(b)] in qualitative agreement with the RSJ model.

From Figs. 1(a) and 1(b) it is clearly seen that the linewidth increases considerably as the temperature is lowered. The RSJ model predicts ${ }^{4}$ that the linewidth varies as

$$
\Delta V_{n}=n^{2} V^{*}\left(R_{a}^{2} / R^{2}\right)\left[1+\frac{1}{2}\left(I_{c} / I\right)^{2}\right] \equiv n^{2} V^{*}\left(R_{e} / R\right)^{2},
$$

where $\Delta V_{n}$ is the $3-\mathrm{dB}$ linewidth on the voltage axis, $V^{*}=4 e k T^{*} R / \hbar$ is the fluctuation voltage, $T^{*}$ is an effective noise temperature, $R$ is the normal-state resistance, $R_{d}$ is the dynamic resistance, $I_{c}$ is the critical current, and $I$ is the dc bias current. The resistance



In Fig. 2 the measured linewidth $\Delta V_{1}$ is plotted as a function of $R_{e}^{2}$. The figure shows that the results approach the dependence given by Eq. (1) at temperatures near the critical temperature (small values of $R_{e}$ ), and the straight line shown in Fig. 2 determines an effective noise temperature $T^{*}=19 \pm 3 \mathrm{~K}$. At low temperatures (large values of $R_{e}$ ) the results deviate markedly from a quadratic dependence on $R_{e}$. Here the dynamic resistance varies considerably across the linewidth and instead of data points we give horizontal bars spanning the range of $R_{e}^{2}$. Also, the variation of $R_{e}$ on the scale of the linewidth is accompanied by an asymmetry of the power spectrum which is, however, only barely visible in Fig. 1(b). This also corroborates that the linewidth is closely correlated with the dynamic resistance, ${ }^{5}$

In previous cavity experiments ${ }^{2,3}$ it was noted that the total power content $P_{1}$ of the first harmonic was propor-

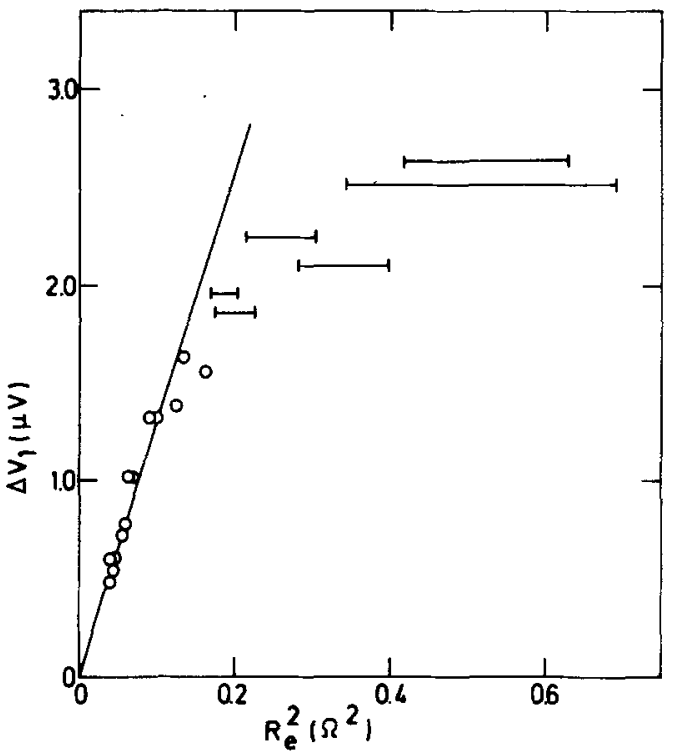

FIG. 2. The measured linewidth at half-peak power $\Delta V_{1}$ as a function of $R_{e}^{2}=R_{d}^{2}\left[1+\frac{1}{2}\left(I_{c} / I^{2}\right]\right.$. The horizontal bars at the large values of $R_{e}$ reflect the observed variation of $R_{d}$ across the linewidth. The initial slope of the curve (corresponding to high temperatures) defines an effective noise temperature $T$ $=19 \pm 3 \mathrm{~K}$ according to Eq. (1).

tional to the critical current squared. As shown in Fig. 3 , where $P_{1}$ is plotted as a function of $I_{c}^{2}$, this is also a reasonable approximation in the present case at least for small values of $I_{c}$. According to the RSJ model, however, the relation $P_{1} \propto I_{c}^{2}$ should apply for large normalized voltages, $V_{1} / R I_{c} \gg 1$. In this and in earlier experiments ${ }^{3}$ we had $V_{1} / R I_{c}<2$ such that we should not be in this limit.

At the lowest temperature $T=3.35 \mathrm{~K}^{7}$ we measured a total emitted power of $5 \times 10^{-12} \mathrm{~W}$ which is about one

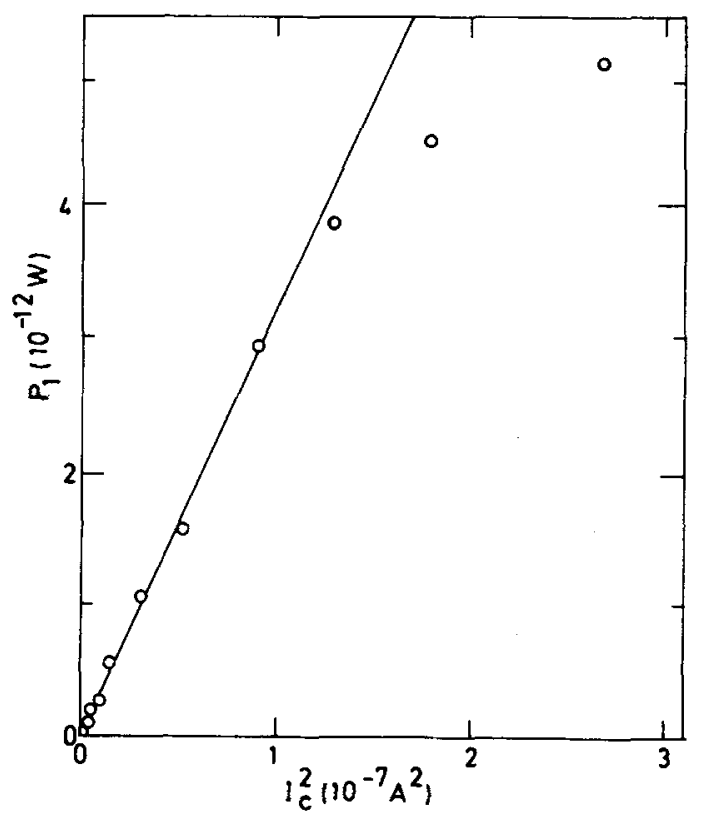

FIG. 3. The measured integral power $P_{1}$ of the first harmonic as a function of the critical current squared. 
order of magnitude larger than previously obtained from superconducting microbridges. ${ }^{2,3}$ The corresponding peak value $4 \times 10^{-14} \mathrm{~W}$ in the $10-\mathrm{MHz}$ receiver bandwidth is, however, of the same order of magnitude as obtained from similar microbridges mounted in a cavity. ${ }^{3}$ With our nonresonant system the larger total power is distributed within a much wider spectral line $\left(\Delta V_{1, \max } \approx 2.6 \mu \mathrm{V}\right.$ compared to $\left.\sim 0.6 \mu \mathrm{V}\right)$ in the cavity experiment. ${ }^{3}$ The maximum power which may be delivered into a $6.5-\Omega$ load resistor is $7 \times 10^{-11} \mathrm{~W}$, estimated on the basis of the RSJ model and using weaklink parameters corresponding to our lowest temperature. A comparison to the experimental value $5 \times 10^{-12}$ W shows that a good coupling between the bridge and the transformer has been obtained. By applying a known external signal to the transformer and measuring the bridge response we have estimated a coupling efficiency only a factor of 10 less than optimum.

For the second harmonic, $n=2$, we can make few conclusions because the signal-to-noise ratio is rather poor. The linewidth increases with increasing $R_{d}$, although the increase is slower than for $n=1$. However, in the region of the $n=2$ peak, $\left(V_{2} \approx 10 \mu \mathrm{V}\right)$ the differential resistance $R_{d}$ decreases with the temperature in contradiction to the RSJ model. This is a well-established deviation ${ }^{4}$ and has been tentatively explained by relaxation processes ${ }^{8,9}$ and/or by electric-field-induced changes of the energy spectrum of the excited quasiparticles in the weak-link region. ${ }^{10}$ It should be noted that the nonresonant impedance matching used here for measuring Josephson radiation from the autonomous junction may be preferable to a cavity system also in mic rowave studies of these nonequilibrium processes.

We have demonstrated that detailed measurements of the spectral distribution of the microwave power generation in autonomous superconducting microbridges may be made. It is crucial to avoid resonances in the experimental setup since such resonances may interact with the sample and strongly modify its properties. We have realized nonresonant conditions using a broadband waveguide transformer to match the waveguide impedance to the junction. We were not able to obtain an ideal impedance match; nevertheless, the coupling efficiency was about an order of magnitude better than previously obtained with cavity coupling schemes. In a narrow temperature interval we followed the variation in line shape of the Josephson radiation over about one decade in linewidth where we, with a cavity system under otherwise similar conditions, had observed a variation in linewidth of at most $50 \%$.

The authors would like to thank Ole Eg for the sample preparation and $\mathrm{L}_{\circ}$ Olsen and $\mathrm{B}$. Krogh for valuable technical assistance. Finally, one of the authors (V. $N_{\circ} G_{\circ}$ ) acknowledges The Danish Ministry of Education for financial support.

${ }^{1}$ A.H. Dayem and C.C. Grimes, Appl. Phys. Lett. 9, 47 (1966).

${ }^{2}$ V.N. Gubankov, V.P. Kosheletz, and G.A. Ovsyannikov, JETP Lett. 21, 226 (1975); Proc. Appl. Supercond. Conf., 1976 (unpublished)

${ }^{3}$ N.F. Pedersen, O.H. Soerensen, J. Mygind, P.E. Lindelof, M. T. Levinsen, and T.D. Clark, Appl. Phys. Lett. 28, 562 (1976).

${ }^{4}$ A.N. Vystavkin, V.N. Gubankov, L.S. Kuzmin, K. K. Likharev, V.V. Migulin, and V.K. Semenov, Rev. Phys. Appl.9, 80 (1974).

${ }^{5} \mathrm{It}$ should be noted that the line shape does depend on whether the spectral distribution is plotted versus detector frequency or dc voltage. This difference becomes important for large values of the linewidth but is not significant in the present experiment.

${ }^{6}$ P. E. Gregers-Hansen, M.T. Levinsen, and G. Fog Pedersen, J. Low Temp. Phys. 7, 99 (1972); P.E. GregersHansen and M. T. Levinsen, Phys. Rev. Lett, 27, 847 (1971).

${ }^{7}$ The experiment could not be extended to lower temperatures because of the appearance of instabilities in the $I-V$ curve at the first harmonic peak.

${ }^{8}$ K. K. Likharev and L. A. Yakobson, IEEE Trans. Magn. MAG-11, 860 (1975).

${ }^{9} \mathrm{H}$. Højgaard Jensen and P.E. Lindelof, J. Low Temp. Phys. 23,469 (1976).

${ }^{10}$ L. G. Aslamasov and A.I. Larkin, Zh. Eksp. Teor. Fiz. 70, $1340(1976)$. 